Document downloaded from:

http://hdl.handle.net/10251/117074

This paper must be cited as:

Jimenez-Meneses P.; Bañuls Polo, M.; Puchades, R.; Maquieira Catala, A. (2018). Fluorthiol Photocoupling Reaction for Developing High Performance Nucleic Acid (NA) Microarrays. Analytical Chemistry. 90(19):11224-11231.

https://doi.org/10.1021/acs.analchem.8b00265

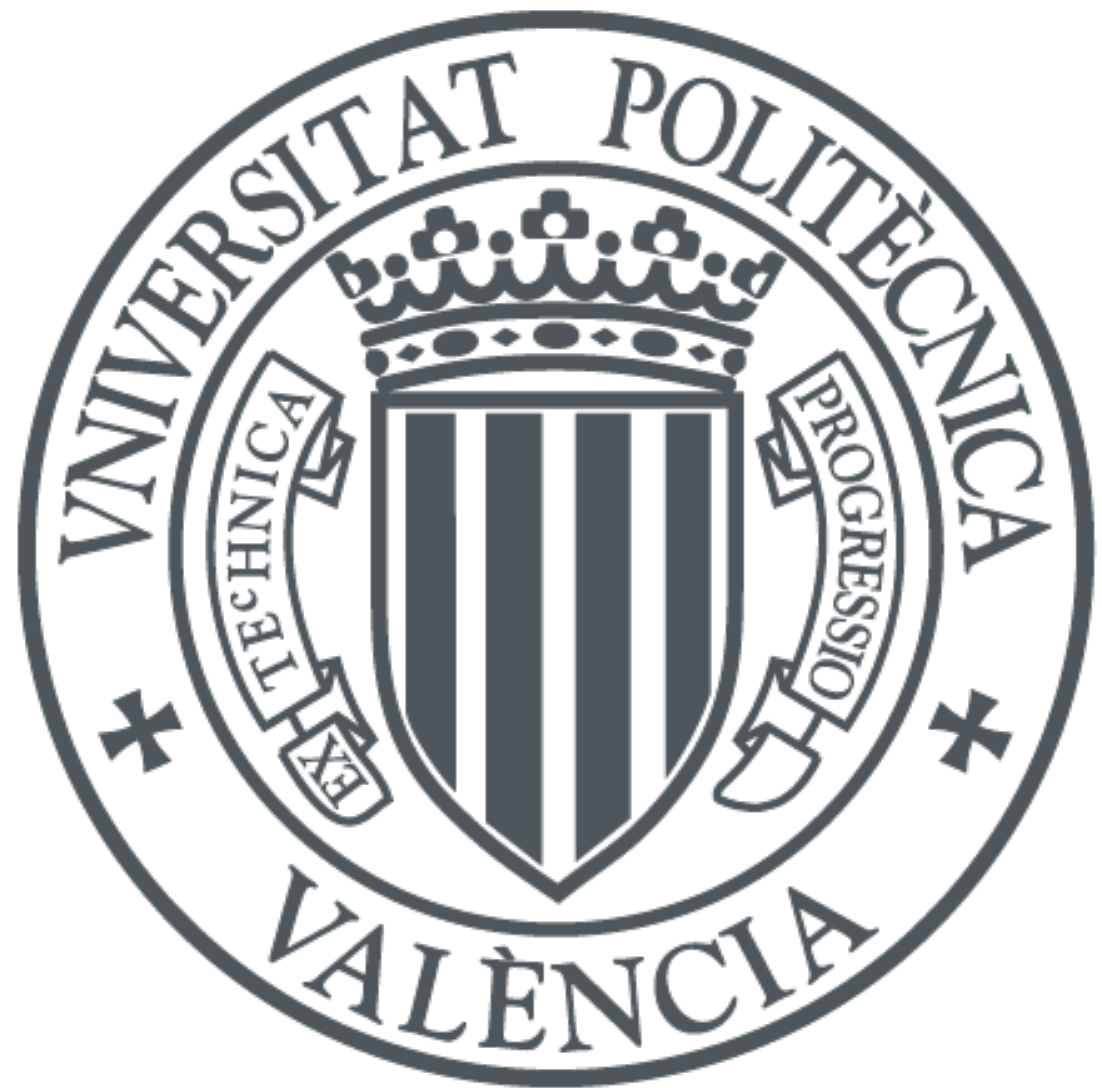

The final publication is available at

http://doi.org/10.1021/acs.analchem.8b00265

Copyright American Chemical Society

Additional Information 


\title{
Fluor-thiol photocoupling reaction for developing high performance nucleic acid (NA) microarrays
}

\author{
Pilar Jiménez-Meneses, María-José Bañuls*, Rosa Puchades, Ángel Maquieira* \\ Departamento de Química, Instituto Interuniversitario de Investigación de Reconocimiento Molecular y Desarrollo Tecnoló- \\ gico (IDM) Universitat Politècnica de València, Universitat de València, Camino de Vera s/n, 46022 Valencia, Spain. \\ mbpolo@upv.es +34 963877342
}

\begin{abstract}
Spatially controlled anchoring of NA probes onto microscope glass slides by a novel fluor-thiol coupling reaction is performed. By this UV-initiated reaction, covalent immobilization in very short times (30 seconds at $254 \mathrm{~nm}$ ) is achieved with probe densities of up to $39.6 \mathrm{pmol} / \mathrm{cm}^{2}$. Modulating the surface hydrophobicity by combining a hydrophobic silane and a hydrophilic silane allow the fabrication of tuned surfaces where the analyte approaches only to the anchored probe, which notably reduces nonspecific adsorption and the background. The generated substrates have proven clear advantages for discriminating single-base-pair mismatches, and for detecting bacterial PCR products. The hybridization sensitivity achieved by these high-performance surfaces is about 1.7 pM. Finally, this anchoring reaction is demonstrated using two additional surfaces: polytetrafluoroethylene (PTFE) and polyvinylidene fluoride (PVDF) membranes. This provides a very interesting pathway for anchoring thiolated biomolecules onto surfaces with C-F motifs via a quick clean UV reaction.
\end{abstract}

Nowadays, the development of new biosensors to diagnose and prevent different illnesses in specifically, selectively and quickly using inexpensive devices is still required. NA microarrays have revolutionized basic research in molecular and cellular biology, biochemistry and genetics. ${ }^{1-3}$ Although the technology in NA is most advanced, protein microarrays also emerge as a potential and useful tool., ${ }^{4}$

During the fabrication of NA microarrays, glass microscope slides are widely used as a support because of their good optical properties, high resistance and rigidity, low cost, and surface chemical derivatization by organosilane chemistry. ${ }^{2}$ Effective surface activation and functionalization protocols are key for microarray production. A good control of these processes improves the performance and quality of assays. For instance, the properties of functionalized surfaces will define efficient immobilization of probes and their biorecognition capacity.

Regarding the immobilization process, covalent anchoring of NA probes is preferred to adsorption because of its robustness and reliability. ${ }^{6,7}$ Besides, photoactivated reactions like fluorthiol photocoupling chemistry that is herein reported, offer several advantages, such as that associated with click chemistry reactions. These reactions are fast, clean and enviro-friendly, and allow oriented immobilization ${ }^{8,9}$ in aqueous media, which is crucial for their bioutility. ${ }^{10,11}$ When light activates the reaction, additional advantages, such as site-specific immobilization, come into play. One example of these photo-click-chemistry reactions that has been recently applied to microarray technology is thiol-ene coupling, ${ }^{12-15}$ which allows biomolecules to be anchored to silicon-based surfaces selectively under mild conditions, with quantitative or near-quantitative yields.

Another important parameter to take into account in either the immobilization or the hybridization process is the surface. Superhydrophobic surfaces are based on nonreactive and low-surface energy functional groups which prevent most chemical and biological substances from adhering. ${ }^{16}$ Thanks to this good property, hydrophobic surfaces can be applied to develop NA microarrays with a water-repellent background that confines the sample only to the point where the probe is anchored by reducing nonspecifity and improving sensitivity. Nevertheless, poor surface wettability can hamper proper contact between the groups on the surface and the probes in the solution by lowering ${ }_{18}$ immobilization yields and hindering effective hybridization. ${ }^{17}$,

Although the use of perfluorinated surfaces is challenging, it can be very useful for applications in microarray technology, among others ${ }^{19-22}$ In fact in this study we report the fluor-thiol photocoupling reaction to covalently bond thiolated probes directly to a perfluoroalkyl surface for the first time. This methodology allows the probes bearing a thiol group to be anchored to the C-F bond of highly hydrophobic surfaces quickly and cleanly. To support the nature of the formed bond, two additional surfaces (PVDF and PTFE membranes) containing C-F motifs are used apart from silanized glass slides.

Given the extreme hydrophobicity of this surface, which hinders effective hybridization, modulating surface hydrophobicity by an optimal combination of paramount water-repellent silane as $1 \mathrm{H}, 1 \mathrm{H}, 2 \mathrm{H}, 2 \mathrm{H}$-perfluorodecyltriethoxysilane (PFTS) and a hydrophilic repellent silane, such as 2-[methoxy(polyethyleneoxy)6-9propyl]trimethoxysilane) (PEGS), is necessary in the functionalization process.

The new developed surface favors the selective approach of the target solution only at the point to which the probe is anchored as it is the most hydrophilic area of the entire surface. This fact minimizes the background and nonspecific binding without having to resort to a blocking step before the hybridization assay. Following this strategy, DNA microarrays can be applied to discriminate single nucleotide polymorphisms (SNPs) and to detect bacterial DNA (e.g. Salmonella). SNPs are 
the most abundant form of genetic variation in the human genome ${ }^{23}$ and allow disease predispositions and drug responses to be predicted, ${ }^{24}$ whereas Salmonella bacteria have been the main cause of foodborne bacterial illnesses in humans in many countries for at least more than 100 years. ${ }^{25}$

\section{EXPERIMENTAL SECTION}

Chemicals, reagents and buffers. The glass microscope slides used as substrates for the microarrays were obtained from Labbox Labware, S.L. (Spain). The Immobilon-P PVDF membranes were acquired from Merck (Spain). The PTFE membranes came from Wolfpack (Spain). 2-[Methoxy(polyethyleneoxy $)_{6-9}$ propyl]trimethoxysilane was purchased from Gelest (Germany). 1H,1H,2H,2H-Perfluorodecyltriethoxysilane and vinyltrimethoxysilane were supplied bySigma-Aldrich (Spain). Toluene was purchased from Scharlau (Spain). 3,3',5,5'-Tetramethylbenzidine liquid substrate was acquired from SDT (Germany). Note: all the chemicals were handled following the corresponding material safety data sheets. All the chemicals were used without further purification.

Milli-Q water, with a resistivity above $18 \mathrm{~m} \Omega$, was used to prepare the aqueous solutions. The employed buffers, phosphate buffer saline (PBS1x, $0.008 \mathrm{M}$ sodium phosphate dibasic, $0.002 \mathrm{M}$ sodium phosphate monobasic, $0.137 \mathrm{M}$ sodium chloride, $0.003 \mathrm{M}$ potassium chloride, $\mathrm{pH}$ 7.5), PBS-T (PBS1x containing $0.05 \%$ Tween 20 ) and saline sodium citrate (SSC1x, $0.15 \mathrm{M}$ sodium chloride, $0.02 \mathrm{M}$ sodium citrate, $\mathrm{pH}$ 7) were filtered through a $0.45-\mu \mathrm{m}$ pore size nitrocellulose membrane of the Fisher brand (Germany) before being used.

Table 1. Used oligonucleotides sequences

\begin{tabular}{|c|c|c|c|}
\hline Name & Sequence (5' to 3') & 5'- & 3'- \\
\hline Probe 1 & $\begin{array}{l}\text { CCCGATTGACCAGCTAGCA } \\
\text { TT- }(\mathrm{T})_{15}\end{array}$ & Cy5 & SH \\
\hline Probe 2 & $\begin{array}{l}(\mathrm{T})_{15^{-}} \\
\text {CCCGATTGACCAGCTAGCATT }\end{array}$ & $\mathrm{SH}$ & - \\
\hline Probe 3 & $\begin{array}{l}(\mathrm{T})_{15^{-}} \\
\text {CCCGATTGACC } \underline{T G C T A G C A T T ~}\end{array}$ & $\mathrm{SH}$ & - \\
\hline Probe 4 & $\begin{array}{l}(\mathrm{T})_{15^{-}} \\
\text {CCCGATTGATTAGCTAGCATT }\end{array}$ & $\mathrm{SH}$ & - \\
\hline Probe 5 & $\begin{array}{l}(\mathrm{T})_{15^{-}} \\
\text {CCATATTGACCAGCTA므ATT }\end{array}$ & $\mathrm{SH}$ & - \\
\hline Probe 6 & $\begin{array}{l}\text { CGCCGATAACTCTGTCTCTG } \\
\text { TA }\end{array}$ & $\mathrm{SH}$ & - \\
\hline Probe 7 & $\begin{array}{l}\text { TTTTGATTACAGCCGGTGTA } \\
\text { CGACCCT }\end{array}$ & $\mathrm{SH}$ & - \\
\hline Probe 8 & $\begin{array}{l}\text { TTTTAGACGCAATACCGCG } \\
\text { AGGTGGAGCA }\end{array}$ & $\mathrm{SH}$ & \\
\hline Probe 9 & $\begin{array}{l}\text { TTTTGATTACAGCCGGTGTA } \\
\text { CGACCCT }\end{array}$ & $\mathrm{SH}$ & Dig \\
\hline A & $\begin{array}{l}\text { AATGCTAGCTGGTCAATCG } \\
\text { GG }\end{array}$ & $A x^{b}$ & - \\
\hline
\end{tabular}

a Digoxigenin, b Alexa Fluor ${ }^{\circledR} 647$

The oligonucleotides in Table 1 were acquired from Eurofins Genomic (Ebersberg, Germany). DNA concentration and quality were determined by measuring optical density at 260/280 $\mathrm{nm}$ in a NanoDrop ND 1000 Spectrophotometer (Thermo Fisher Scientific, Wilmington, Delaware, USA). The PCR- amplified Salmonella products were obtained as previously described. $^{26,27}$

Instrumental methods. Contact angle system OCA20, equipped with the SCA20 software, was provided by Dataphysics Instruments GmbH (Filderstadt, Germany). Measurements were taken in quintuplicate at room temperature with a volume drop of $5 \mu \mathrm{L}$ employing the $18 \mathrm{~m} \Omega$ water quality.

Microarray printing was done with a low-volume noncontact dispensing system from Biodot (Irvine, CA, USA), model AD1500. Irradiation at $254 \mathrm{~nm}$ was carried out in a UV-Ozone Surface Cleaner (UVOH 150 LAB; FHR, Anlagenbau, Germany, GMBH), while irradiation at $365 \mathrm{~nm}$ was performed with a mercury capillary lamp (6 $\mathrm{mW} / \mathrm{cm}^{2}$, Jelight Irvine, CA, USA) placed at a fixed distance $(0.5 \mathrm{~cm})$.

The fluorescence signal of the spots was recorded with a homemade surface fluorescence reader (SFR) operating with a high-sensitive charge couple device camera Retiga EXi (Qimaging Inc., Burnaby, Canada) with light-emitting diodes Toshiba TLOH157P as the light source. ${ }^{28}$ A microarray scanner Axon4000B (Molecular Devices, CA, USA) was employed for the immobilization density determinations. The GenePix Pro 4.0 software (Molecular Devices, Inc. Sunnyvale, CA, USA) was utilized for the microarray image analysis and subsequent quantification.

X-ray photoelectron spectra were recorded with a Sage 150 spectrophotometer (SPECS Surface Nano Analysis GmbH, Berlin, Germany). Nonmonochromatic Al Ka radiation (1486.6 $\mathrm{eV}$ ) was used as the X-ray source operating at $30 \mathrm{eV}$ constant pass energy for the elemental specific energy binding analysis. The vacuum in the spectrometer chamber was $9 \times 10^{-9} \mathrm{hPa}$ and the analyzed sample area was $1 \mathrm{~mm}^{2}$. Attenuated total reflectance infrared spectra were recorded by a Bruker Tensor 27 FTIR coupled to a Platinum ATR accessory.

Silanization of slides. Commercial glass slides were cut into chips $(2 \times 1 \mathrm{~cm})$ and were cleaned with a UV-Ozone Surface Cleaner for $7 \mathrm{~min}$ at $254 \mathrm{~nm}$ to remove organic contaminants. To introduce the reactive functional groups, these chips were immersed in a $2 \% \mathrm{v} / \mathrm{v}$ solution of the corresponding silane in toluene for $45 \mathrm{~min}$ at room temperature. Then samples were withdrawn from the silane solution, washed several times with toluene and air-dried. Next chips were baked for $1 \mathrm{~h}$ at $110^{\circ} \mathrm{C}$. To generate the hydrophobic surfaces, chips were treated with $1 \mathrm{H}, 1 \mathrm{H}, 2 \mathrm{H}, 2 \mathrm{H}$-perfluorodecyltriethoxysilane (surface A), whereas a combination of 2-[methoxy(polyethyleneoxy) 6 -9propyl] trimethoxysilane and $1 \mathrm{H}, 1 \mathrm{H}, 2 \mathrm{H}, 2 \mathrm{H}$-perfluorodecyltriethoxysilane was used at a ratio of $4: 1 \mathrm{v} / \mathrm{v}$ (surface B) for the optimized surfaces. The control substrates were functionalized with vinyltrimethoxysilane (surface C), and with 2-[methoxy(polyethyleneoxy) 6-9propyl] (surface D), as explained before.

Oligonucleotide immobilization. The attachment of the thiolated and nonthiolated oligonucleotides onto different surfaces was studied. On the one hand, glass microscope slides were treated following the above-described procedure to obtain the corresponding surface A, B and D. To prove covalent anchoring, Probe 1 consisting of a 5'-Cy5- and 3'-SH-labeled oligonucleotide, and Target A consisting of a 5'-AlexaFluor ${ }^{\circledR 647-}$ labeled oligonucleotide (Table 1), were spotted at 0.5, 1 and 2 $\mu \mathrm{M}$ in PBS1x onto surfaces A and D, and were exposed to UVlight at $254 \mathrm{~nm}$ for $30 \mathrm{~s}$ to induce the immobilization. Afterward slides were thoroughly rinsed with SSC1x and air-dried. On the other hand, the PVDF and PTFE membranes were used as supports with no further modification. Probe 1 and Target $\mathrm{A}$ at 0.5 , 
1 and $2 \mu \mathrm{M}$ in PBS1x were spotted onto both surfaces and exposed to UV-light at $254 \mathrm{~nm}$ for $5 \mathrm{~s}$ to encourage immobilization. Afterward substrates were thoroughly rinsed with PBS-T and air-dried. Finally, in order to calculate immobilization density, increasing concentrations of Probe 1 (from 0.01 to $10 \mu \mathrm{M}$; $40 \mathrm{~nL} /$ spot; 4 spots/row) in PBS1x were spotted onto surface B and exposed to UV-light at $254 \mathrm{~nm}$ for $30 \mathrm{~s}$ to produce immobilization. Slides were thoroughly rinsed with SSC1x and airdried. The immobilization results were obtained from the fluorescence signals using a microarray scanner.

Hybridization Assays. To study the hybridization efficiency on the developed surface, glass chips were treated as described above to obtain surface B. Afterward the solutions containing serial dilutions of Probe 2 (from 0.25 to $5 \mu \mathrm{M}$ ) in PBS1x were spotted $(40 \mathrm{~nL})$ onto surface B to create the microarray (four spots per concentration). Then slides were exposed to UV-light at $254 \mathrm{~nm}$ for $30 \mathrm{~s}$, washed with SSC1x and air-dried. After washing, $15 \mu \mathrm{L}$ of Target A (concentrations ranging from 0.0005 to $0.25 \mu \mathrm{M}$ in SSC1x) were spread over the surface with a coverslip. After incubation in a humid chamber for $1 \mathrm{~h}$ at 37 ${ }^{\circ} \mathrm{C}$, the coverslip was gently removed, and the chip was washed with SSC1x. The fluorescence intensity of the spots was recorded using the SFR as before.

Mismatches detection. Four oligonucleotide sequences, Probes 2, 3, 4 and 5 having zero, one, two and three base mismatches for Target A, respectively, and Probe 6 as the negative control (no complementary) were spotted $(0.5 \mu \mathrm{M}, 40 \mathrm{~nL} / \mathrm{spot})$ with a noncontact dispenser onto surface B to create the microarray. After probe immobilization, the microarray was subjected to hybridization with Target A (50 nM) in SSC under different astringency conditions (salt concentration from $0.1 \mathrm{x}$ to $5 \mathrm{x}$ and formamide content from $0-25 \%$ ) for $1 \mathrm{~h}$ at $37^{\circ} \mathrm{C}$. After washing and drying, fluorescence was measured with the SFR.

Detection of the Salmonella PCR products. Glass slides were perfluoro-functionalized as described above. Then the solutions containing SH-labeled Probe 7 (the Salmonella-specific probe), Probe 8 (the negative hybridization control) and Probe 9 (the positive development control) at 1 and $2 \mu \mathrm{M}$ were spotted in PBS1x onto surface B to fabricate the microarrays. Afterward slides were exposed to UV-light at $254 \mathrm{~nm}$ for $30 \mathrm{~s}$ and subsequently washed with SSC1x and air-dried. Then the digoxigenin-labeled PCR product solutions $(15 \mu \mathrm{L})$ in hybridization buffer (SSC1x) were distributed over the chip. PCR duplexes were first denaturated by a 10 -minute incubation at $95^{\circ} \mathrm{C}$ followed by fast cooling for $1 \mathrm{~min}$ on ice. After incubating $1 \mathrm{~h}$ at $37{ }^{\circ} \mathrm{C}$, slides were washed with SSC1x and air-dried. Finally, slides were incubated with an HRP-labeled anti-digoxigenin antibody produced in goat (dilutions ranging from $1 / 10$ to $1 / 10000$ in $\mathrm{SSC} 1 \mathrm{x}$ and $15 \%$ of formamide) for $30 \mathrm{~min}$ at room temperature, washed with SSC1x, air-dried and revealed with TMB solution for around 2 min.

\section{RESULTS AND DISCUSSION}

Surface Chemical Derivatization. Surface functionalization was studied to achieve a reproducible protocol to fabricate homogenous and stable surfaces that allowed the covalent anchoring of NA probes and the hybridization process. First, glass microscope slides were cleaned with a UV-ozone lamp for 7 minutes to provide the high density of the hydroxyl groups on the surface, which are key for effective silanization. Then substrates were functionalized by immersion in organosilane solu- tion (2\% in toluene). Given the potential difficulties of the microarray in the later hybridization process due to the high hydrophobicity of perfluorinated surfaces (surface A), different derivatizations to modulate surface wettability were studied. The proper combination of an excellent water repellent as PFTS, with a highly hydrophilic and anti-fouling compound as PEGS, could allow tuned surfaces to be obtained on which the analyte approaches specifically to the appropriate immobilized probe, thus preventing nonspecific adsorption and lowering the background.

In an attempt to achieve a commitment between the surface repellence and bioavailability of the anchored probes, the PEGS-PFTS ratio and functionalization times were optimized. After several experiments to modulate surface hydrophobicity, functionalization times of 45 minutes and a volume ratio of 1:4 (PFTS/PEGS) showed an optimal contact angle (about $100^{\circ}$ ) and, thus, promising surfaces for successful hybridization. Longer times or higher PFTS concentrations were not suitable for the hybridization process because of the excessive surface hydrophobicity, which lowered the surface wettability too much by impeding the target solution to properly reach the anchored probes. Thus, every substrate was functionalized for $\mathbf{4 5}$ minutes at room temperature to acquire the corresponding surface properties, and hydrophobicity was controlled to ensure reproducible and homogeneous surfaces.

Six different surfaces were used in this study: first, a highly hydrophobic surface only made of PFTS to prove the fluor-thiol reaction (surface A). Second, a wettability-tuned surface with an optimal PFTS-PEGS ratio (1:4) to facilitate the hybridization process (surface B). Third, a control standard surface was functionalized with vinyltrimethoxysilane (surface $\mathrm{C})^{29}$ in comparison to surface B. Fourth, an anti-fouling control surface using organosilane PEGS (surface D) was prepared. Finally, two additional surfaces (PVDF and PTFE membranes) were used to support the anchoring mechanism. These surfaces did not require further activation or functionalization, which cut the assay time.

The quality of the surface was monitored by thoroughly measuring the water contact angle (WCA) on the whole surface. As seen in Figure S1, the commercial glass slides displayed a contact angle of $37^{\circ}$, whereas the activated surfaces showed contact angles around $0^{\circ}$ by the UV-lamp. This is attributed to an increased number of hydroxyl moieties generated on the surface by the oxidation and cleaning treatment. Upon functionalization with the corresponding organosilanes, hydrophobic surface A, with a WCA of $110^{\circ}$, and modulated surface B, with a WCA of around $100^{\circ}$, were obtained. Surfaces C and D provided a WCA of around $70^{\circ}$ and $27^{\circ}$, respectively. The raw PVDF and PTFE membranes displayed a contact angle of 130 ${ }^{\circ}$ and $148^{\circ}$, respectively.

The X-ray photoelectron spectroscopy (XPS) experiments with the raw glass slides, and with surfaces $\mathrm{A}$ and $\mathrm{B}$, were performed. The emergence of $\mathrm{CF}_{2}$ and $\mathrm{CF}_{3}$ peaks at 290 and 292 $\mathrm{eV}$ on the functionalized surfaces corroborated the success of the functionalization process (Figure S2). The chemical composition of modulated surface B was established from high-resolution XPS as a part of the surface characterization (Table S1).

DNA immobilization assays. Based on the paper of Al-Gharabli et al. who established C-F cleavage in the presence of an oxidizing agent, ${ }^{30}$ immobilization of a thiolated probe for its reactivity with perfluorinated surfaces under UV irradiation was 
investigated. This is an unknown reaction with an excellent potential in microarray technology, among others, inspired in the thiol-para fluor "click" chemistry. In this reaction, the para-F position in the aromatic ring showed high reactivity to nucleophilic substitution. ${ }^{19-21}$ Thus in order to demonstrate the use of the fluor-thiol photochemical reaction for tethering thiolated DNA, a 5' thiol-ended probe, labeled with Cy5 at its 3' end (Table 1 , Probe 1), was spotted at different concentrations in PBS1x (from 0.01 to $4 \mu \mathrm{M} ; 40 \mathrm{~nL} /$ spot; 4 spots/row) onto surface $A$, and displayed successful fluorescence intensity after thorough washing.

Several irradiation times (from a few seconds to $1 \mathrm{~h}$ ) and two wavelengths (365 and $254 \mathrm{~nm}$ ) were investigated. The best results in fluorescence intensity terms were obtained for UV exposure times of 30 seconds at $254 \mathrm{~nm}$. This time noticeably reduced those previously reported to create DNA microarrays by thiol-ene and thiol-yne photo click reactions, where times of at least 20 minutes, and $365 \mathrm{~nm}$ are used. ${ }^{11,13,29,31}$ This difference was due to the low-power irradiation of the $365 \mathrm{~nm}$ lamp (6 $\mathrm{mW} / \mathrm{cm}^{2}$ ) compared to the current $254 \mathrm{~nm}$ lamp $\left(50 \mathrm{~mW} / \mathrm{cm}^{2}\right)$, and was also due to the change of wavelength itself from 365 $\mathrm{nm}$ to $254 \mathrm{~nm}$, the latter of which is more energetic irradiation.

This novel immobilization has a clear advantage over the published work as regards irradiation times, and is an interesting technique to be applied to many fields, like nanoparticles biofunctionalization.

Several assays were run to corroborate the linking nature and to prove the reliability of the system.

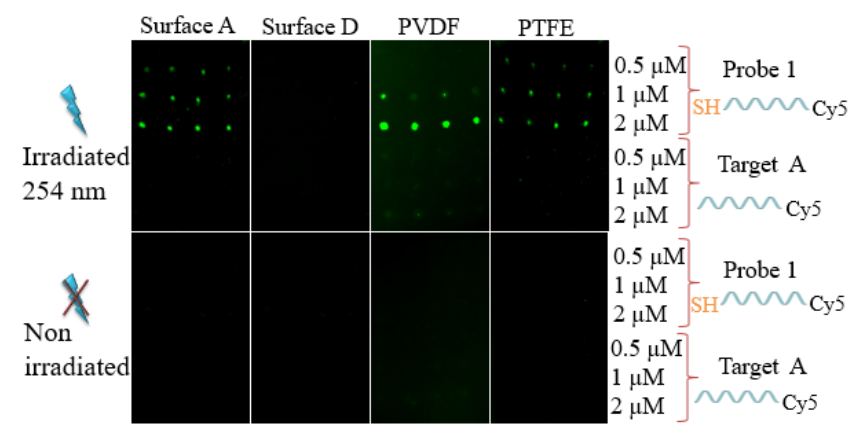

Figure 1. The labeled thiolated and nonthiolated probes were spotted at different concentrations onto surfaces A, D, PVDF and PTFE. Afterward, surfaces were irradiated. Fluorescence images were recorded, after washing, by SFR. The control surfaces without irradiation were also measured after the washing steps.

First, immobilization studies on surfaces A and D were performed (Figure 1). Surfaces were spotted with the fluorescencetagged thiolated and non thiolated probes (Probe 1 and Target A, respectively) at different concentrations (from 0.5 to $2 \mu \mathrm{M}$ ). Afterward, surfaces were exposed for 30 seconds to UV-light at $254 \mathrm{~nm}$, or were kept in dark, and washed. Then, the fluorescence of the microarray was recorded with the SFR. Successful thiolated probe immobilization onto surface A occurred after irradiation, whereas the nonthiolated probe did not anchor. The thiolated and nonthiolated probes did not adsorb onto surface A when kept in the dark without irradiation. Surface D showed the expected anti-bioadsorption capability, and the thiolated and nonthiolated probes were not immobilized after irradiation. Therefore, we conclude that a thiol motif and UV irradiation are necessary to achieve covalent bonding to surface A, and suggest the formation of a reactive thiol radical.
Second to identify the anchoring moiety on the surface, the above protocol was applied to two additional surfaces: PVDF and PTFE (Figure 1). With surface A, three anchoring points were possible; bonds Si-O, the $\mathrm{C}-\mathrm{H}$ and $\mathrm{C}-\mathrm{F}$. Thus, the PVDF membranes that only had C-H and C-F motifs were used. Different irradiation times, from $1 \mathrm{~s}$ to $1 \mathrm{~min}$, were assayed. The assays showed that a 5-second photoexposure were enough to achieve successful probe immobilization. Regarding the results, the attachment of the thiolated probe to this surface continued to occur, whereas the nonthiolated probe did not anchor to the surface. Moreover without irradiation, no fluorescence signal was detectable. These results ruled out the bonding between the thiol and the silane, and left open the possibility of nucleophilic substitution or dehydrohalogenation taking place. To this end, the same experiments on the PTFE surface - $\left(\mathrm{CF}_{2}-\mathrm{CF}_{2}\right)_{\mathrm{n}}$ - were performed. These surfaces, irradiated with the thiolated probes for only 30 seconds, showed successful probe immobilization with high-fluorescence intensity after thorough washing. Additionally, the nonirradiated surfaces showed nofluorescence, as well as the nonthiolated probes. These results support a covalent attachment between the thiol and the $\mathrm{C}$ of the $\mathrm{C}-\mathrm{F}$ bonds, with $\mathrm{F}$ being displaced as it was the only common motif in all the substrates.

Third, X-ray photoelectron spectroscopy (XPS) analyses were done to know the nature of the chemical bonding between a thiolated compound and the perfluorinated surfaces (Figure 2). Surface A was irradiated for 1 minute at $254 \mathrm{~nm}$ under the UV lamp in presence of a thiolated probe and washed, and compared with the raw surface A. The contribution of the C-C bond increased dramatically and a new contribution from $\mathrm{COO}$ bonds was observed. All this pointed to the attachment of the probe to the surface. It should be noted that the profile of the C1s peak did not vary significantly when the raw surface was irradiated in the absence of the thiolated compound (Figure S2). The $\mathrm{CF}_{3} / \mathrm{CF}_{2}$ ratio remained unchanged for all the control surfaces and only changed in the case of the surface A irradiated in presence of thiolated probe, demonstrating the role of C-F bonds in the probe attachment.
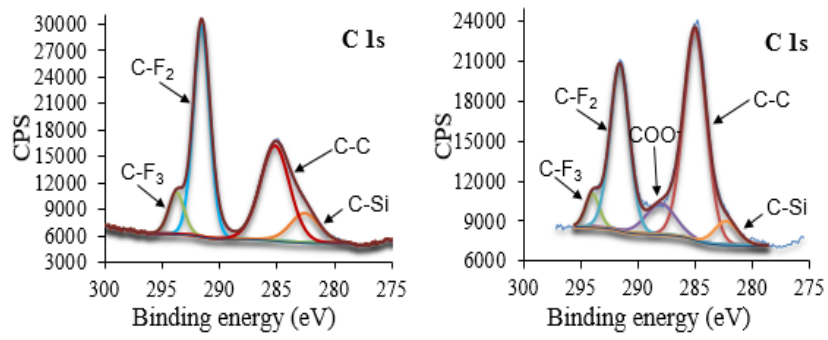

Figure 2. XPS high-resolution spectra. Left. C 1s spectrum of surface A. Right C 1s spectrum of surface A + thiolated compound + UV irradiation and washing. C-F $F_{2}$ bond at $291 \mathrm{eV}$ and $\mathrm{C}-\mathrm{F}_{3}$ bond at $293 \mathrm{eV}$.

Finally, the ATR-IR experiments on the PVDF membranes were performed. This surface was chosen for its composition to allow very thin layers to be better detected. No changes in the glass slides and Teflon surfaces were detected using these instruments. Microscope glass slides are too wide compared to the thin thiolated compound layer formed. With the Teflon membranes, the C-F signals of the anchored compound matched the C-F peaks of the membrane itself. Thus $40 \mu \mathrm{L}$ of $1 \mathrm{H}, 1 \mathrm{H}, 2 \mathrm{H}, 2 \mathrm{H}$-perfluorodecanethiol $0.90 \mathrm{M}$ in methanol were added to a PVDF membrane $(1 \times 1 \mathrm{~cm})$. After solvent evaporation, membranes were irradiated for 1 minute at $254 \mathrm{~nm}$ under 
the UV lamp. Afterward, membranes were washed with methanol for 30 minutes to ensure the complete removal of the nonanchored compound. After washing thoroughly, the C-F signals of the thiolated compound remained. However, no significant spectral change was observed after washing when the membrane was not irradiated in the presence of the thiolated compound. This corroborates a covalent attachment between the thiolated compound and the PVDF surface. Figure 3 shows the FTIR-spectra obtained for the raw and treated membranes, where new peaks appear and are attributed to the aliphatic $-\mathrm{CF}_{2}$ (1000-1150 $\left.\mathrm{cm}^{-1}\right)$ and $-\mathrm{CF}_{3}\left(1350-1100 \mathrm{~cm}^{-1}\right)$ stretchings, and, more importantly, to the aliphatic CF stretching $\left(1100-1000 \mathrm{~cm}^{-}\right.$ ${ }^{1}$ ), which supports the bonding of the thiol compound to the $\mathrm{CF}_{2}$ moieties of the surface.

Therefore, we report that, upon irradiation, the formed radical thiols attack the $\mathrm{C}$ of a $\mathrm{CF}_{2}$ unit by displacing the $\mathrm{F}$ of the substrate, and shows the anchoring of a thiolated probe to a C-F bond for the first time (Figure S3).

Immobilization of $1 \mathrm{H}, 1 \mathrm{H}, 2 \mathrm{H}, 2 \mathrm{H}$-perfluorodecanethiol onto PVDF membranes

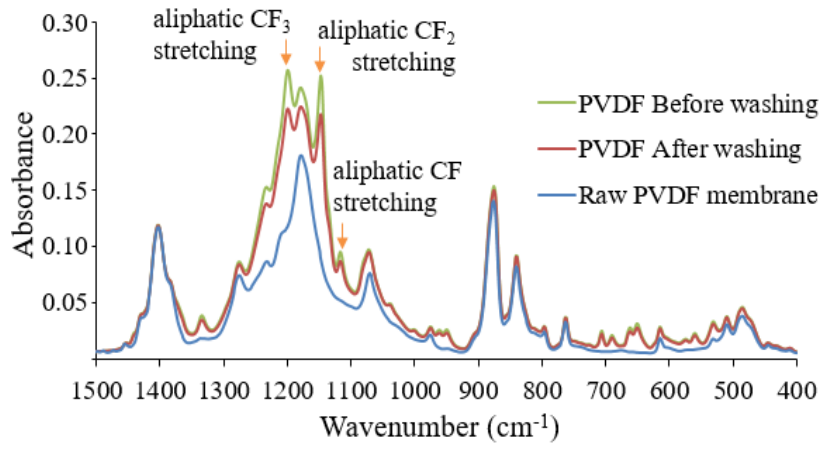

Figure 3. ATR-FTIR spectra. Blue. Raw PVDF membrane. Green. PVDF membrane irradiated in the presence of $1 \mathrm{H}, 1 \mathrm{H}, 2 \mathrm{H}, 2 \mathrm{H}-\mathrm{per}-$ fluorodecanethiol before washing. Red. PVDF membrane irradiated in the presence of $1 \mathrm{H}, 1 \mathrm{H}, 2 \mathrm{H}, 2 \mathrm{H}$-perfluorodecanethiol after washing.

Having demonstrated the fluor-thiol photo-click reactions, and knowing that the low wettability of these surfaces can hinder successful hybridization, immobilization efficiency and further hybridization on surface B were studied. As explained above, the wettability of surface B was tuned by a combination of two different repellent organosilanes to provide an optimal WCA of $100^{\circ}$. Thus a standard calibration curve was constructed by spotting Probe 1 in PBS1x onto surface B (from 0.01 to $10 \mu \mathrm{M}$ ), irradiating 30 seconds with the UV-surface cleaner and recording fluorescence with a microarray scanner. After washing, fluorescence was once again recorded and the resulting intensity was interpolated to the calibration curve to determine immobilization density (Figure S4). Under the studied conditions, surface saturation was reached for Probe 1 at $4 \mu \mathrm{M}$ with an immobilization density of $39.6 \mathrm{pmol} / \mathrm{cm}^{2}$ (Figure 4). Higher concentrations did not lead to any increment in fluorescence intensity. These values were around 8-fold higher than those reported by the thiol-ene coupling reaction on the vinyltrimethoxysilane functionalized surfaces (C). ${ }^{13}$ Moreover, surface B led to smaller and reproducible spot sizes, and higher fluorescence intensities than surface $\mathrm{C}$, which implies better performance for the new surfaces. These results support the potential application of a fluorinated surface to construct high-density NA microar- rays given that the covalent anchoring of thiolated oligonucleotides to aliphatic fluorinated surfaces by photoactivation is herein demonstrated. Taking into account that the maximum packing density of double-stranded DNA is around $3 \times 10^{13}$ molecules $/ \mathrm{cm}^{2}$ (i.e., $50 \mathrm{pmol} / \mathrm{cm}^{2}$ ), ${ }^{32}$ our surfaces came close to a maximal feasible surface immobilization.
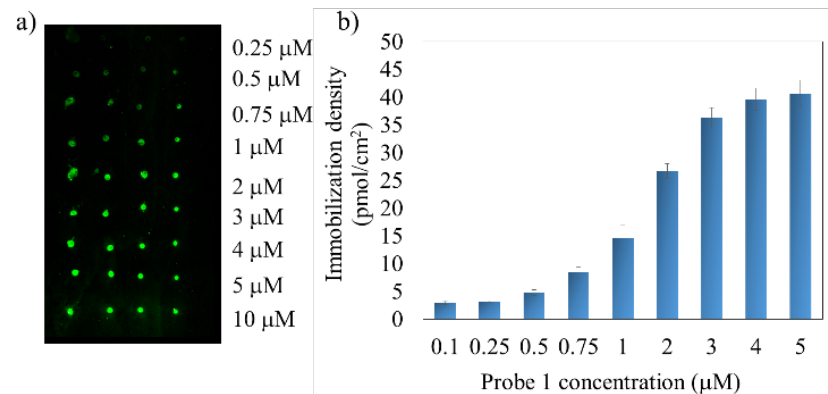

Figure 4. (a) Fluorescence image of an array with Probe 1 immobilized at different concentrations and (b) the oligonucleotide immobilization densities obtained for Probe 1 vs. the spotted concentration. These values were obtained from the interpolation on the corresponding calibration curve. The error bars where obtained from 3 chips, with 4 spots per concentration for each chip.

DNA hybridization assays. To prove the capability of surface B to improve the detection of complementary strands in a sensitive and selective way, hybridization assays were carried out. Serial dilutions of 3' $\mathrm{SH}$ nonlabeled Probe 2 (from 0.25 to 5 $\mu \mathrm{M}, \mathrm{PBS} 1 \mathrm{x}$ ) were immobilized onto surface $\mathrm{B}$ as optimized before, and hybridization with AlexaFluor647-labeled fully complementary strand, Target 1 , at different concentrations (from 0.0005 to $0.25 \mu \mathrm{M}$ in SSC1x), was performed. After incubation in a humid chamber for $1 \mathrm{~h}$ at $37^{\circ} \mathrm{C}$ and washing, the fluorescence intensity of the spots was recorded by the SFR. The obtained fluorescence intensity against the Target A concentration is plotted in Figure 5 for each assayed concentration of Probe 2. The hybridization signal increased with

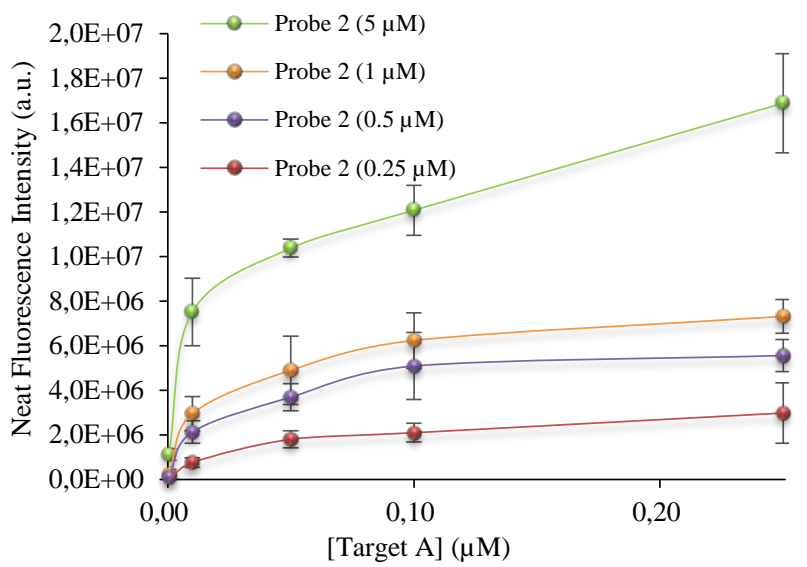

Figure 5. Neat fluorescence intensity vs. concentration of Target A for different concentrations of Probe 2. The error bars where obtained from 2 chips, with 4 spots per concentration for each chip. 


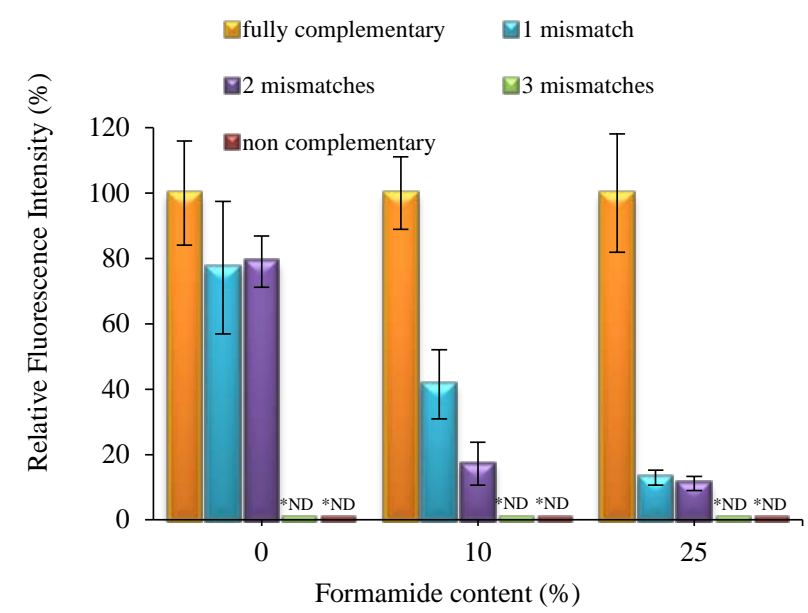

Figure 6 . The relative fluorescence intensity signals obtained for fully complementary, 1-3 mismatches, and noncomplementary probes at $0.5 \mu \mathrm{M}$ in PBS1x by hybridizing with Target $\mathrm{A}$ at 0.05 $\mu \mathrm{M}$ in SSC0.1x to display different formamide contents for the analysis of SNPs on surface B. *ND not detectable. The error bars where obtained from 3 chips, with 4 spots per concentration for each chip.

target concentration, with up to $0.5 \mathrm{nM}$ detected, which corroborates the suitability of these microarrays to detect hybridized oligonucleotide sequences at very low levels. This excellent sensitivity was attributed to the hydrophobicity modulation, a small spot size and the high immobilization density obtained by the proposed approach. Identical experimental conditions were used on surface $C$ for comparison reasons. The lowest detected Target A concentration improved the value obtained with control surface $\mathrm{C} 2$-fold and, with previous reported work onto alkynyl surfaces, ${ }^{11}$ it fell within the order of the estimated limit of detection, for the target, and the detection device used for these experiments (calculated as the concentration providing the blank signal, plus three times its standard deviation). Here, it should be noticed that the ionic strength used (SSC1x) is low to overcome the electrostatic repulsion due to the high probe coverage achieved. But, the hydrophobic environment surrounding the probe spots concentrates the target solution uniquely on the hydrophilic areas where the probes are attached. Thus, water evaporation leads to a selective concentration of target molecules and salts on the probe spots, improving the efficiency of the hybridization.

Hybridization assays with Target A were replicated to determine the intrachip and interchip relative standard deviations (RSD). Intrachip RSD ranged from $6 \%$ to $11 \%$, whereas interchip RSD varied from $9 \%$ to $16 \%$. Unlike most of the reported methods in DNA microarrays, a blocking step was not necessary, which simplified the process and reduced times. ${ }^{33}$

Having demonstrated the successful hybridization process, which proves the wide applicability of these DNA microarrays, patterned surfaces were used to discriminate single nucleotide polymorphisms (SNPs) and to detect bacterial PCR amplification products with high sensitivity and selectivity.

In the medical field, the use of SNPs assays allows the influence of different factors to be studied in disease susceptibility and responses to drugs. ${ }^{34}$ The ability to differentiate SNPs in DNA assays is most important in selectivity terms. ${ }^{23,35}$ Therefore, a base mismatch study was performed by immobilizing five 5' $\mathrm{SH}$ modified probes at $0.5 \mu \mathrm{M}$ in PBS1x (containing from zero to three mismatches and an additional noncomplementary probe) onto surface B. Afterward, substrates were subjected to hybridization with a labeled target, complementary to the zero mismatches probe, at $50 \mathrm{nM}$ using different salt concentrations (SSC from $0.1 \mathrm{x}$ to $5 \mathrm{x}$ ) and formamide content (from $0 \%$ to $25 \%$ ). Fluorescence was measured by SFR after washing and drying chips. The fully complementary probe displayed the highest fluorescence intensity, while the other probes clearly showed lower fluorescence. A higher formamide concentration and a lower salt concentration made the hybridization process difficult, which rendered it more specific (Figure S5). Using astringency conditions (SSC0.1x, 25\% formamide), the results revealed a clear discrimination between the fully complementary probe and all the probes (see Figure 6). The signals obtained for one mismatch and the fully complementary probes were differentiated by $85 \%$, while three mismatches and noncomplementary probes did not exhibit any detectable signal. The results indicate that under the described conditions, the microarray is quite able to distinguish a single base mutant sequence at a very good sensitivity level.

The early detection of pathogens, i.e. bacteria strains, in water or food is paramount to maintain good human and animal safety. In our case, as proof of concept, the optimized substrates (surface B) were used to detect PCR products from an innocuous serotype of Salmonella bacteria. A specific nucleotide sequence (Probe 7, 1 and $2 \mu \mathrm{M}$ ), complementary to the central region of a 152 bp amplicon, was immobilized as before. Additional probes, used as a negative hybridization control (Probe 8) and a positive development control (Probe 9), were also included in the microarray. Hybridization with the digoxigeninlabeled PCR product solutions (denaturated and cooled with ice previously), incubation with anti-digoxigenin HRP-labeled antibody produced in goat, and development with 3,3',5,5'-tetramethylbenzidine (TMB) substrate, were carried out.

As seen in Figure 7, the spots corresponding to probe 7 show a dark precipitate, whereas no precipitation happened in the negative controls (Probe 8), which indicates the specificity of the hybridization process. Under the reported conditions, the generated DNA microarray was able to detect up to $1.7 \mathrm{pM}$ of Salmonella amplification products. These figures were 75 -fold lower than those reported in the literature for vinyl-functionalized surfaces, where thiolated probes were attached by thiol-ene coupling chemistry. ${ }^{29}$ These results corroborate the good performance of our approach to determine genomic DNA at very low levels. Thus combining light-induced probe anchoring with the modulation of surface hydrophobicity properties, an advanced surface material for microarraying was designed.

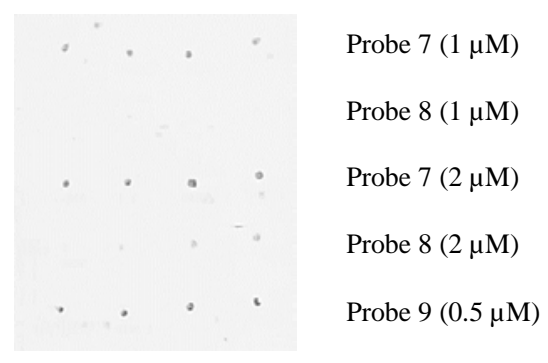

Figure 7. The absorbance signal obtained for the selective recognition of the PCR-amplified DNA samples of Salmonella using specific capture Probe $7(1$ and $2 \mu \mathrm{M})$, Probe $8(1$ and $2 \mu \mathrm{M})$ as the 
nonspecific hybridization control, and Probe $9(0.5 \mu \mathrm{M})$ as the development control. Biochip after development read by a document scanner.

\section{CONCLUSIONS}

The fluor-thiol photocoupling reaction has been demonstrated in heterogeneous medium for the first time using non aromatic fluoride compounds and light as the catalyst. The reaction was successfully applied to prepare DNA microarrays by probe covalent immobilization in a reproducible and oriented manner onto glass microscope slides, PVDF and PTFE membranes as substrates. These surfaces are very useful for the design and development of DNA biosensors as they allow the specific confinement of the analyte solution to the anchored probe sites.

The reported immobilization process is fast, compatible with aqueous media and enviro-friendly. Moreover, like the elevated surface hydrophobicity, platforms showed a high density of probes at the interface, which avoids nonspecific adsorption and significantly reduces the background. Thanks to its simplicity, quickness and use of green chemistry, this route is very promising for the immobilization of thiolated oligos onto any support that involves C-F motifs (e.g. perfluoro-functionalized slides, PVDF and PTFE membranes).

The optimized surfaces showed the maximum immobilization density achievable for a DNA self-assembled monolayer. The wettability modulation of the microscope glass slides through specific functionalization allowed robustness, stability and optimal surface conditions for later DNA probe strand hybridization. This improved by 2-fold the limit of quantification compared to a control surface and to previous works.

As demonstrated, the surface properties modulation and the application of the fluor-thiol coupling reaction enabled to perform interesting applications, like discriminating single nucleotide polymorphisms and the sensitive detection of PCR amplified DNA products, and gave better results compared to conventional methods.

From these results, we offer a very interesting pathway to develop diverse detection devices with a heterogeneous format. Moreover, linking chemistry can be applied to the biofunctionalization of nanoparticles where, for instance, repellent properties would help to avoid aggregation.

\section{ASSOCIATED CONTENT}

\section{Supporting Information}

Water contact angles, XPS analysis, mechanism proposed for the coupling reaction, calibration curve for Target A and hybridization assays done under different conditions to discriminate single nucleotide polymorphisms (Word)

\section{AUTHOR INFORMATION}

\section{Corresponding Author}

*E-mail: mbpolo@upvnet.upv.es

\section{ORCID}

María-José Bañuls: 0000-0002-2422-7731

\section{Author Contributions}

The manuscript was written with contributions from all the authors, who all approved the final version of the manuscript

\section{ACKNOWLEDGMENTS}

Financial support from INTERBOINTER (project CTQ201345875-R) and BIHOLOG (Project CTQ2016-75749-R), FEDER and GVA PROMETEO II 2014/040 is acknowledged. The authors also thank Dr. Tortajada-Genaro and Dr. Niñoles Rodenes for providing the Salmonella and Campylobacter PCR-amplified products. P. J.-M. acknowledges the Spanish Ministry of Economy, Industry and Competitiveness for the public FPI grant (Project CTQ2013-45875-R) and the co-financing by the European Social Fund. Dr. Miguel Ángel González-Martínez, Dr. Sergio Navalón and Dr. Patricia Concepción from Universitat Politècnica de València are acknowledged for their help in the XPS analysis.

\section{REFERENCES}

(1) Schena, M.; Shalon, D.; Davis, R. W.; Brownt, P. Quantitative Monitoring of Gene Expression Patterns with a Complementary DNA Microarray. Science 1995, 270, 467-470. (1)

(2) Pilarsky, C.; Nanaduri, L. K.; Roy, J. Gene Expression Analysis in the Age of Mass Sequencing. In Cancer Gene Profiling: Methods and Protocols, Methods in Molecular Biology; Grutzmann, R., Pilarsky, C., Eds.; Springer Science+Business Media New York 2016, 2016; pp 67-73.

(3) Gorreta, F.; Carbone, W.; Barzaghi, D. Genomic Profiling: cDNA Arrays and Oligoarrays. In Molecular Profiling: Methods and Protocols, Methods in Molecular Biology; Virginia Espina and Lance A. Liotta, Ed.; Springer Science+Business Media, 2012; pp 89-105.

(4) Gupta, A.; Mishra, A.; Puri, N. Peptide Nucleic Acids: Advanced Tools for Biomedical Applications. J. Biotech. 2017, 259, 148159.

(5) Duarte, J. G.; Blackburn, J. M. Advances in the development of human protein microarrays. Expert Rev. Proteomics 2017, 14, 627641.

(6) Singh, V.; Zharnikov, M.; Gulino, A.; Gupta, T. DNA Immobilization, Delivery and Cleavage on Solid Supports. J. Mater. Chem. 2011, 21, 10602-10618.

(7) Lockett, M. R.; Phillips, M. F.; Jarecki, J. L.; Peelen, D.; Smith, L. M. A Tetrafluorophenyl Activated Ester Self-Assembled Monolayer for the Immobilization of Amine-Modified Oligonucleotides. Langmuir 2008, 24, 69-75.

(8) Kolb, H. C.; Finn, M. G.; Sharpless, K. B. Click Chemistry: Diverse Chemical Function from a Few Good Reactions. Angew. Chem. Int. Ed. 2001, 40, 2004-2021.

(9) Evans, R. A. The Rise of Azide-Alkyne 1,3-Dipolar "click" Cycloaddition and Its Application to Polymer Science and Surface Modification. Aust. J. Chem. 2007, 60, 384-395.

(10) Dondoni, A. The Emergence of Thiol-Ene Coupling as a Click Process for Materials and Bioorganic Chemistry. Angew. Chem. Int. Ed. 2008, 47, 8995-8997.

(11) Escorihuela, J.; Bañuls, M.-J.; Puchades, R.; Maquieira, Á. Site-Specific Immobilization of DNA on Silicon Surfaces by Using the Thiol-yne Reaction. J. Mater. Chem. B 2014, 2, 8510-8517.

(12) Hoyle, C. E.; Bowman, C. N. Thiol-Ene Click Chemistry. Angew. Chem. Int. Ed. 2010, 49, 1540-1573.

(13) Escorihuela, J.; Bañuls, M. J.; Grijalvo, S.; Eritja, R.; Puchades, R.; Maquieira, Á. Direct Covalent Attachment of DNA Microarrays by Rapid Thiol-Ene "Click" Chemistry. Bioconjug. Chem. 2014, 25, 618-627.

(14) Escorihuela, J.; Bañuls, M. J.; Puchades, R.; Maquieira, Á. DNA Microarrays on Silicon Surfaces through Thiol-Ene Chemistry. Chem. Commun. 2012, 48, 2116-2118.

(15) Gupta, N.; Lin, B. F.; Campos, L. M.; Dimitriou, M. D.; Hikita, S. T.; Treat, N. D.; Tirrell, M. V; Clegg, D. O.; Kramer, E. J.; Hawker, C. J. A Versatile Approach to High-Throughput Microarrays Using Thiol-Ene Chemistry. Nat. Chem. 2010, 2, 138-145.

(16) Vargo, T. G.; Calvert, J. M.; Chen, M.-S.; Gardella, J. A.; Jeffrey Calvert, J. M. Jeffrey Calvert, J. M. Adhesive Electroless Metallization of Fluoropolymeric Substrates. Science 1993, 262, 17111712 . 
(17) Li, J.; Li, L.; Du, X.; Feng, W.; Welle, A.; Trapp, O.; Grunze, M.; Hirtz, M.; Levkin, P. A. Reactive Superhydrophobic Surface and Its Photoinduced Disulfide-Ene and Thiol-Ene (Bio)Functionalization. Nano Lett. 2015, 15, 675-681.

(18) Zhou, H.; Wang, H.; Niu, H.; Gestos, A.; Wang, X.; Lin, T. Fluoroalkyl Silane Modified Silicone Rubber/Nanoparticle Composite: A Super Durable, Robust Superhydrophobic Fabric Coating. Adv. Mater. 2012, 24, 2409-2412.

(19) Becer, C. R.; Babiuch, K.; Pilz, D.; Hornig, S.; Heinze, T.; Gottschaldt, M.; Schubert, U. S. Clicking Pentafluorostyrene Copolymers: Synthesis, Nanoprecipitation, and Glycosylation. Macromolecules 2009, 42, 2387-2394.

(20) Slavin, S.; Burns, J.; Haddleton, D. M.; Becer, C. R. Synthesis of Glycopolymers via Click Reactions. Eur. Polym. J 2011, 47, 435446.

(21) Wild, A.; Winter, A.; Hager, M. D.; Görls, H.; Schubert, U. S. Perfluorophenyl-Terpyridine Ruthenium Complex as Monomer for Fast, Efficient, and Mild Metallopolymerizations. Macromol. Rapid Commun. 2012, 33, 517-521.

(22) Boyer, C.; Davis, T. P. One-Pot Synthesis and Biofunctionalization of Glycopolymers via RAFT Polymerization and Polymerization and Thiol - Ene Reactions. Chem. Commun. 2009, 40, 6029-6031.

(23) Banoub, J. H.; Newton, R. P.; Esmans, E.; Ewing, D. F.; Mackenzie, G. Recent Developments in Mass Spectrometry for the Characterization of Nucleosides, Nucleotides, Oligonucleotides, and Nucleic Acids. Chem. Rev. 2005, 105, 1869-1915.

(24) Mccarthy, J. J.; Hilfiker, R. The Use of Single-Nucleotide Polymorphism Maps in Pharmacogenomics. Nat. Biotechnol. 2000, 18, 505-508.

(25) Crump, J. A.; Gordon, M. A.; Parry, C. M. Epidemiology, Clinical Presentation, Laboratory Diagnosis, Antimicrobial Resistance, and Antimicrobial Management of Invasive Salmonella Infections. Clin. Microbiol. Rev. 2015, 28, 901-937.

(26) Arnandis-Chover, T.; Morais, S.; Tortajada-Genaro, L. A.; Puchades, R.; Maquieira, Á.; Berganza, J.; Olabarria, G. Detection of food-borne pathogens with DNA arrays on disk. Talanta 2012, 101, 405-412.

(27) Santiago-Felipe, S.; Tortajada-Genaro, L. A.; Morais, S.; Puchades, R.; Maquieira, Á. Isothermal DNA amplification strategies for duplex microorganism detection. Food Chem. 2015, 174, 509-515.

(28) Mira, D.; Llorente, R.; Morais, S.; Puchades, R.; Maquieira, A.; Marti, J. High Throughput Screening of Surface-Enhanced Fluorescence on Industrial Standard Digital Recording Media. Proc. SPIE 2004, 5617, 364-373.

(29) Bañuls, M.-J.; Jiménez-Meneses, P.; Meyer, A.; Vasseur, J.J.; Morvan, F.; Escorihuela, J.; Puchades, R.; Maquieira, A. Improved Performance of DNA Microarray Multiplex Hybridization Using Probes Anchored at Several Points by Thiol-Ene or Thiol-Yne Click Chemistry. Bioconjug. Chem. 2017, 28, 496-506.

(30) Al-Gharabli, S.; Kujawa, J.; Mavukkandy, M. O.; Arafat, H. A. Functional Groups Docking on PVDF Membranes: Novel Piranha Approach. Eur. Polym. J. 2017, 96, 414-428.

(31) González-Lucas, D.; Bañuls, M. J.; Puchades, R.; Maquieira, Á. Versatile and Easy Fabrication of Advanced Surfaces for High Performance DNA Microarrays. Adv. Mater. Interfaces 2016, 3, 1500850.

(32) Georgiadis, R., Peterlinz K. P., and Peterson, A. W. Quantitative Measurements and Modeling of Kinetics in Nucleic Acid Monolayer Films Using SPR Spectroscopy. J. Am. Chem. Soc. 2000, 122, 3166-3173.

(33) Bammler T, Beyer RP, Bhattacharya S, Boorman GA, Boyles A, Bradford BU, Bumgarner RE, Bushel PR, Chaturvedi K, Choi D, Cunningham ML, Deng S, Dressman HK, Fannin RD, Farin FM, Freedman JH, Fry RC, Harper A, Humble MC, Hurban P, Kavanagh TJ, Kaufmann WK, K, Z. H. M. of the T. R. C. Standardizing Global Gene Expression Analysis between Laboratories and across Platforms. Nat. Methods 2005, 2, 351-356.

(34) Ostrem, J. M.; Peters, U.; Sos, M. L.; Wells, J. a; Shokat, K. M. K-Ras(G12C) Inhibitors Allosterically Control GTP Affinity and Effector Interactions. Nature 2013, 503, 548-551.

(35) Wang, K., Tang, Z., Yang, C. J., Kim, Y., Fang, X., Li, W., Wu, Y.; Medley, C. D., Cao, Z., Li, J., Colon, P., Lin, H., and Tan, W. Molecular Engineering of DNA: Molecular Beacons. Angew. Chem. Int. Ed. 2009, 48, 856-870. 
For TOC only:

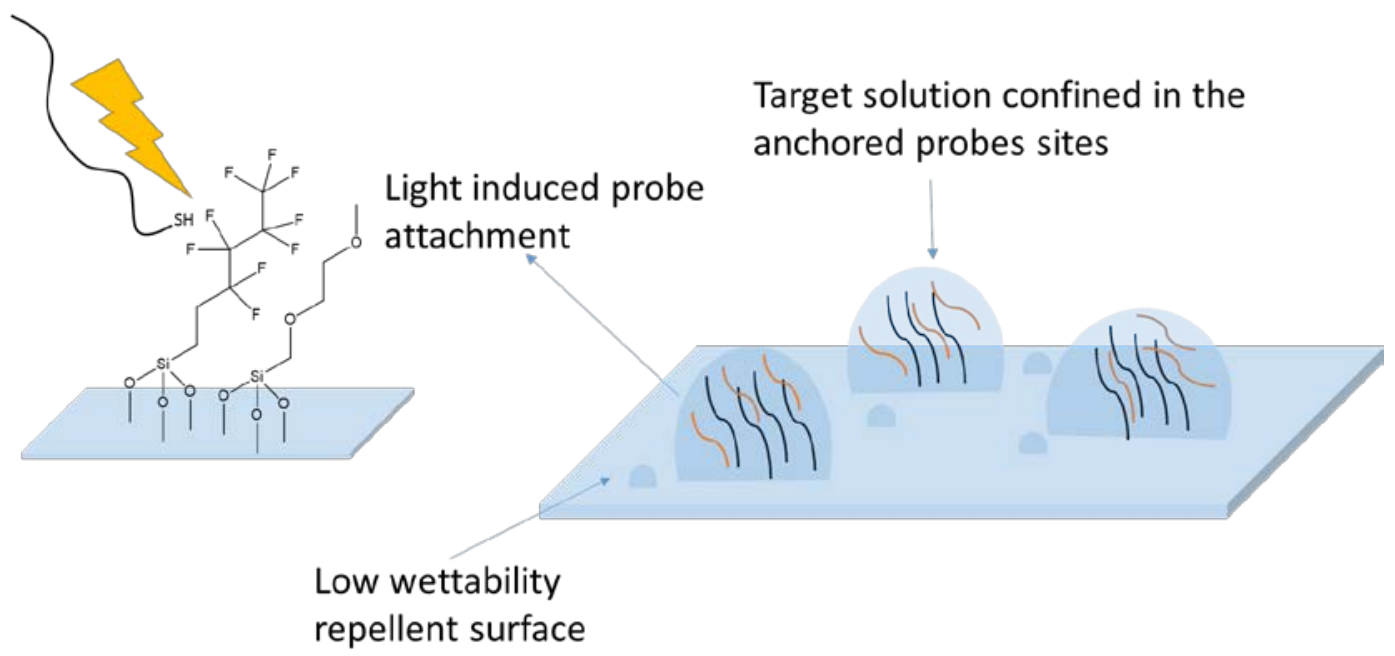

